\title{
Evaluation and screening for hereditary renal cell cancers
}

\author{
W. Marston Linehan, M.D.
}

Urologic Oncology Branch, National Cancer Institute Bethesda, Maryland

See related article on page 319.

Cite as: Can Urol Assoc J 2013;7(9-10):324-5. http://dx.doi.org/10.5489/cuaj.1685

Published online October 7, 2013.

$\mathrm{R}$ eaume and colleagues present a thoughtful guide to genetic screening for inherited renal cancers, including von Hippel Lindau (VHL), hereditary papillary renal cell carcinoma (HPRC), Birt-Hogg-Dubé (BHD), tuberous sclerosis complex (TSC) and hereditary paraganglioma/ pheochromocytoma. ${ }^{1}$ Our experience has been similar. For patients with familial clear cell renal cancer, we recommend $V H L$ germline mutation testing for those who have either a family history of VHL or a VHL clinical phenotype (i.e., bilateral renal cysts/tumours, pancreatic neuroendocrine tumours, retinal angiomas, CNS hemangioblastomas, etc.). However, in familial renal clear cell patients without a VHL clinical phenotype, we recommend germline karyotypic analysis (to evaluate for the presence of chromosome 3 translocations) or SDHC germline mutation testing. To date, we have not detected a germline $V H L$ gene mutation in a patient/family with clear cell "kidney only" manifestations.

For patients at risk for hereditary papillary renal carcinoma, we recommend germline MET mutation testing starting at age 21. HPRC is, in general, a late onset disease; however, we have identified an early onset MET mutation in which kidney tumours have been detected in patients as young as 19 years old. ${ }^{2}$ While bilateral multifocal type 1 papillary RCC is common, hereditary papillary renal carcinoma is truly rare; less than 30 families are known to exist. Germline MET mutations are rarely identified in patients with bilateral, multifocal type 1 papillary RCC with no family history.

The initial diagnosis of Birt-Hogg-Dubé is most often made by clinical findings. In $90 \%$ of our BHD families, an individual was found to have cutaneous fibrofolliculoma, while $84 \%$ of patients were found to have pulmonary cysts, and nearly $30 \%$ had a history of pneumothorax. Although the

BHD-associated kidney cancer pathologic phenotype can be variable (mostly hybrid/oncocytic RCC, chromophobe RCC and clear cell RCC), nearly $60 \%$ of tumours are hybrid/ oncocytic renal cell carcinoma. We recommend germline FLCN testing, beginning at age 21 , in families with patients with cutaneous fibrofolliculomas, familial pulmonary cysts/ pneumothorax and/or those found to have a hybrid/oncocytic RCC pathology.

Hereditary leiomyomatosis and renal cell carcinoma (HLRCC), which is a hereditary cancer syndrome in which affected individuals are at risk for developing cutaneous and uterine leiomyomas and a potentially aggressive form of type 2 papillary kidney cancer, are characterized by a germline mutation of the gene for the TCA cycle enzyme, fumarate hydratase $(F H)$. We recommend germline $\mathrm{FH}$ mutation testing (starting at 8 years of age) for patients with cutaneous/uterine leiomyomas and/or type II papillary RCC. FH germline mutation testing is important in all patients at risk for HLRCC, as HLRCC-associated kidney cancer has the potential to be an aggressive, lethal form of type II papillary kidney cancer which can spread when the tumours are very small. It is recommended that patients affected with HLRCC undergo annual abdominal MRI imaging, as HLRCCassociated kidney tumours have been detected in patients from 10 to 77 years of age.

Succinate dehydrogenase kidney cancer (SDH-RCC) is another TCA cycle gene hereditary kidney cancer syndrome characterized by germline mutation $S D H B, S D H C$, and $S D H D$. SDH-RCC families can present with kidney cancers, which often have a characteristic "oncocytic" pathologic phenotype; these families can also develop both kidney cancers and pheochromocytomas/paragangliomas. We recommend SDH germline for patients with familial pheochromocytoma and renal cell carcinoma, as well as those with the characteristic SDHB-deficient pathologic pattern.

We also recommend germline mutation testing in early onset (under age 46) kidney cancer, patients with bilateral, multifocal disease and those with a family history of kid- 
ney cancer. ${ }^{3}$ We use clinical phenotypic manifestations (as described in Reaume and colleagues ${ }^{1}$ ), as well as age and kidney cancer pathology as a guide to select which genes to test. For patients with familial clear cell, we consider recommending testing for $V H L, S D H C, B A P 1, T C S 1$ and TSC2. For patients with familial type 1 papillary RCC, we generally recommend germline $M E T$ testing. For patients with familial type 2 papillary RCC, we recommend germline $\mathrm{FH}$ testing. For familial chromophobe RCC, we recommend FLCN, TSC1, TSC2, and sometimes PTEN (depending on the clinical manifestations). For early onset familial RCC, we often recommend $\mathrm{SDH}$ and $\mathrm{FH}$ germline mutations testing. ${ }^{4}$

Acknowledgement: This research was supported by the Intramural Research Program of the National Institutes of Health, National Cancer Institute and the Center for Cancer Research.

\section{References}

1. Reaume MN, Graham GE, Tomiak E, et al. Canadian Guideline on Genetic Screening for Hereditary Renal Cell Cancers. Can Urol Assoc J 2013;7: 319-23. http://dx.doi.org/10.5489/cuaj.1496

2. Schmidt LS, Nickerson ML, Angeloni D, et al. Early onset Hereditary Papillary Renal Carcinoma: germline missense mutations in the tyrosine kinase domain of the Met proto-oncogene. J Urol 2004;172:1256-61. http://dx.doi.org/10.1097/01.ju.0000139583.63354.e0

3. Shuch B, Vourganti S, Middelton L, et al. Defining Early-Onset Kidney Cancer: Implications for Germline and Somatic Mutation Testing and Clinical Management. J Clin Oncol 2013;in Press.

4. Linehan WM. Genetic basis of kidney cancer: role of genomics for the development of disease-based therapeutics. Genome Res 2012;22:2089-100. http://dx.doi.org/10.1101/gr.131110.111

Correspondence: Dr. W. Marston Linehan, Urologic Oncology Branch, National Cancer Institute, 10 Center Drive MSC 1 107, CRC Room 1W-5940, Bethesda, Maryland 20892-1107; WML@nih.gov

Competing interests: None declared. 